\title{
Implications of the 2015 World Health Organization isoniazid preventive therapy recommendations on tuberculosis prevention efforts in Namibia
}

\author{
S A Oloo, MB ChB, MMed \\ Department of Internal Medicine, School of Medicine, University of Namibia, Windhoek, Namibia
}

Corresponding author: S Oloo (adikini84@yahoo.com)

\begin{abstract}
The World Health Organization recently released guidelines recommending 36-month use of isoniazid preventive therapy in adults and adolescents living with HIV in resource-limited settings. Namibia continues to grapple with one of the highest incidences of tuberculosis (TB) worldwide. Implementation of these guidelines requires considerations of TB epidemiology, health infrastructure, programmatic priorities and patient adherence. This article explores the challenges Namibia currently faces in its fight against TB and the implications of the new guidelines on Namibian TB prevention efforts.
\end{abstract}

S Afr Med J 2016;106(8):775-776. DOI:10.7196/SAMJ.2016.v106i8.10787

Isoniazid preventive therapy (IPT) is a key strategy recommended by the World Health Organization (WHO) for the prevention of tuberculosis (TB) in patients infected with HIV. The WHO recently updated its guidelines and now recommends IPT for a duration of at least 36 months for adults and adolescents living with HIV in resource-limited settings. ${ }^{[1]}$ The WHO notes that the implementation of continuous IPT requires considerations of: (i) TB epidemiology; (ii) health infrastructure; (iii) programmatic priorities; and (iv) patient adherence. Individuals with HIV are at an increased risk of developing active TB infection, with studies ${ }^{[2]}$ showing that the incidence of TB doubles within the first year of HIV seroconversion and increases fourfold after 2 years. This article discusses some of the challenges Namibia currently faces in its ongoing fight against TB and the implications of the new WHO IPT recommendations.

\section{Discussion}

Namibia, an upper-middle-income sub-Saharan African country, continues to grapple with what are among the highest prevalences of both HIV and TB worldwide. This, coupled with a sparsely distributed population, presents a great challenge in terms of healthcare delivery.

The incidence of TB in Namibia remains one of the highest in the world, with a case notification rate of 589 cases per 100000 population. ${ }^{[3]}$ In its continued effort to attain its vision of a Namibia where TB is no longer a health threat, ${ }^{[4]}$ the Ministry of Health and Social Services (MoHSS) works very closely with both primary healthcare providers and public health services at national, regional and district levels to ensure the success of the WHO directly observed therapy, short-course (DOTS) strategy in the treatment of TB patients, and to create public awareness of practical interventions for the prevention of TB, especially in people living with HIV. According to the 2012/2013 ${ }^{[5]}$ MoHSS report, the TB and HIV co-infection rate at the time stood at $47 \%$.

A 2014 - 2015 MoHSS report ${ }^{[6]}$ indicates that there has been a small decline in case numbers of TB, suggesting a declining incidence of the disease burden in the country. Significant improvements in TB/HIV service coverage are exemplified by: (i) improved HIV testing for TB patients, currently at $92 \%$ compared with $76 \%$ in 2010 ; and (ii) improved coverage of antiretroviral therapy (ART) among TB/HIV patients, up to $84 \%$ from $43 \%$ in 2010 .
The overall prevalence of HIV in the country is currently estimated at $13.1 \%$ in the general population although there is wide variation in different regions. Despite government efforts to improve ART coverage, Namibia remains one of the top ten countries in the world worst affected by HIV, which in turn continues to fuel the rise of TB in the country. Over 230000 people aged $>15$ years are living with HIV. ${ }^{[7]}$ The number of people living with HIV is expected to increase as ART coverage increases, reducing the numbers of AIDS-related deaths.

\section{Health infrastructure and cost implications}

The structure of the health sector in Namibia aims to decentralise health services as much as possible while integrating public health strategies in service delivery. The continued collaboration of the Namibia TB Leprosy Program with the National Institute of Pathology and the Division of Pharmaceutical Services has demonstrated outstanding success in ensuring the timely provision of quality laboratory services, as well as uninterrupted supply of TB drugs to the TB treatment centres in both the capital city and remote clinics in the rural areas.

However, in health facilities in the peripheral regions, including the border towns, services are still grossly inadequate, especially in terms of human resources. Studies ${ }^{[8]}$ in the Omaheke region show that more service-related factors than community-related factors influenced the successful implementation of community-based TB care. According to the MoHSS, ${ }^{[6]}$ the TB delivery programme in this region was inadequate, with a $51.4 \%$ defaulter rate.

In its health budget planning, the government of Namibia needs to consider the projected cost implications of adopting the new 36-month IPT strategy as opposed to a 6-month IPT strategy. Smith et al..$^{[9]}$ examined the cost of implementation of IPT in Botswana, where ART is readily available, and found that a 36 -month IPT strategy for HIV-positive individuals was more cost-effective for reducing both $\mathrm{TB}$ and death than individual costs of: (i) providing IPT without tuberculin skin testing (TST); (ii) providing only 6 months of IPT; or (iii) expanding ART eligibility without IPT. Gupta et al. ${ }^{[10]}$ also showed that models providing ART coverage to $90 \%$, TB infection control and 36-month IPT strategy averted most 
TB cases and were more cost-effective than models that provided less ART coverage.

\section{Possible adverse events and the balance between benefit and harm}

The key outcome of interest in the development of the new recommendations was progression to active TB. Other outcomes of interest include adverse effects and adherence. In the formulation of the new guidelines, a meta-analysis of adverse events was not performed because of the heterogeneity of the definition of sideeffects by the different studies that were considered by the WHO. The study by Swaminathan et al. ${ }^{[1]}$ did not report information on adherence, while Martinson et al.'s ${ }^{[12]}$ recorded adherence of $60.4 \%$ in the 36 -month IPT group compared with $83.8 \%$ in the 6 -month IPT group. There is also currently insufficient evidence to indicate whether continuous IPT use increases the risk of development of isoniazid resistance. According to Swaminathan et al. ${ }^{[11]}$ and Samandari et al., ${ }^{[13]}$ the observed proportion of resistance cases among TB cases was similar to the expected rate. However, resistance patterns are known to vary vastly from region to region.

\section{Conclusion}

Implementation of the new IPT strategy in HIV-positive patients would be likely to record success alongside ART strategies, as patients can be counselled for drug adherence and monitored on therapy at the same time. However, this will require a renewed commitment from the government of Namibia and its collaborators to implement the intervention, finance the additional costs associated with drug supply and increase the human resources capacity.

1. World Health Organization. Update 2015 - Recommendations on 36 Months on Isoniazid Preventive Therapy to Adults and Adolescents Living with HIV in Resource-constrained and High TB- and HIVprevalence Settings. Geneva: WHO, 2015. http://www.who.int/tb/publications/2015_ipt_update/en/ (accessed 11 May 2016).

2. Sonnenberg P, Glynn JR, Fielding K, Murray J, Godfrey-Faussett P, Shearer S. How soon after infection with HIV does the risk of tuberculosis start to increase? A retrospective cohort study in South African gold miners. J Infect Dis 2005;15:191(2):150-158. DOI:10.1086/426827

3. Ministry of Health and Social Services, Namibia. Organisation and programme management. In: National Guidelines for the Management of Tuberculosis. 3rd ed. Windhoek: MoHSS, 2012:3-5.

4. Ministry of Health and Social Services, Namibia. Second Medium-Term Strategic Plan for Tuberculosis and Leprosy (2010 - 2015). Windhoek: MoHSS, 2010.

5. Ministry of Health and Social Services, Namibia. The Namibia AIDS Response Progress Report 2015; Reporting Period: 2013 - 2014. Windhoek: MoHSS, 2015.

6. Ministry of Health and Social Services, Namibia. National Tuberculosis and Leprosy Programme (2014 2015). Windhoek: MoHSS, 2015

7. UNAIDS. Namibia HIV and AIDS estimate. http://www.unaids.org/sites/default/files/epidocuments/ NAM.pdf (accessed 12 May 2016)

8. Zvavamwe S, Ehlers VJ. Factors associated with community-based TB care in the Omaheke region, Namibia. Afr J Nurs Midwifery 2007;9(1):59-72

9. Smith T, Samandari T, Abimbola T, Marston B, Sangrujee N. Implementation and operational research: Cost-effectiveness of antiretroviral therapy and isoniazid prophylaxis to reduce tuberculosis and death in people living with HIV in Botswana. J Acquir Immune Defic Syndr 2015;70(3):e84-e93. DOI:10.1097/QAI.0000000000000783

10. Gupta S, Abimbola T, Date A, et al. Cost effectiveness of the three I's for HIV/TB and ART to prevent TB among people living with HIV. Int J Tuberc Lung Dis 2014;18(10):1159-1165. DOI:10.5588/ ijtld.13.0571

11. Swaminathan S, Menon PA, Gopala N, et al. Efficacy of a 6-month versus a 36-month regimen for Swaminathan S, Menon PA, Gopala N, et al. Efficacy of a 6-month versus a 36-month regimen for
prevention of tuberculosis in HIV-infected persons in India: A randomised clinical trial. PloS One prevention of tuberculosis in $\mathrm{HIV}$-infected persons in India: A randomised clinical trial. PloS One

12. Martinson NA, Barnes GL, Moulton LH, et al. New regimens to prevent tuberculosis in adults with HIV infection. N Engl J Med 2011;365(1):11-20. DOI:10.1056/NEJMoa1005136

13. Samandari T, Agizew TB, Nyirenda S, et al. 6-month versus 36-month isoniazid preventive treatment for tuberculosis in adults with HIV infection in Botswana: A randomised, double-blind, placebocontrolled trial. Lancet 2011;377(9777):1588-1598. DOI:10.1016/S0140-6736(11)60204-3

Accepted 30 May 2016. 